\title{
Electroanalytical study of the antidepressant sertraline
}

\author{
Henri P.A. Nouws, Cristina Delerue-Matos, Aquiles A. Barroso, Jose' A. Rodrigues
}

\begin{abstract}
A flow injection square wave cathodic stripping voltammetric method has been developed for the determination of sertraline in a pharmaceutical preparation. The method shows linearity between peak current intensity and sertraline concentration for the interval between $0.20 \times 10^{-6}$ and $1.20 \times 10^{-6} \mathrm{~mol} \mathrm{~L}^{-1}$. Limits of detection and quantification were found to be $1.5 \times 10^{-7}$ and $5.0 \times 10^{-7} \mathrm{~mol} \mathrm{~L}^{-1}$, respectively. Up to 70 samples per hour can be analysed with a good precision (R.S.D. $=2.5 \%$ ). The proposed method was successfully applied to the determination of sertraline in a commercial product. In the voltammetric determination of sertraline in flow, a high sample rate is obtained at reduced costs, opening the possibility to compete with the chromatographic methods generally used for this analysis.
\end{abstract}

Keywords: Flow injection; Hanging mercury drop electrode; Adsorptive stripping voltammetry; Sertraline; SSRI

\section{Introduction}

Sertraline (STR, Fig. 1), a member of the class of the selective serotonin reuptake inhibitors (SSRI), is a compound primarily used as an antidepressant drug but it is also applied in the correction of other psychic deviations like obsessive-compulsive, panic and posttraumatic stress disorders.

For drug development and treatment purposes, it is of high importance to determine the concentration of the drug, and in some cases of its metabolites, in a wide variety of samples (serum, plasma, urine and pharmaceuticals) in order to establish its pharmacokinetics, metabolic pathway, dosage, etc. This implies the need for the development of analytical methodologies, which provide high sample rates, low limits of detection and low maintenance costs.

The determination of STR in serum and plasma is generally performed by high performance liquid chromatography (HPLC) coupled with spectrophotometric detectors, ultraviolet/diode array [1-6] and fluorimetric [7,8]. The wide spread usage of HPLC methods is justified by the high sensitivity and low limit of detection usually obtained and the possibility of the simultaneous analysis of the drug and its metabolites, which increases its usage even further. The main disadvantages of HPLC are the relatively long analysis times and the high acquisition and maintenance costs. Several other methods based on micellar electrokinetic capillary chromatography [9], isotachophoresis [10], gas chromatography-mass spectrometry/nitrogen phosphorus detector [11-15] and UV-vis spectrophotometry [16] have also been described. No assay methods for STR determination are reported in the British, United States and European Union Pharmacopoeias.

Until now, only one publication on the electrochemical study and analysis of STR has been published [17]. This study focused on the determination of the compound in a pharmaceutical product using square wave adsorptive stripping voltammetry under batch conditions.

Besides its wide spread use in metal analysis, adsorptive stripping voltammetry is also adequate for the analysis of organic compounds, which can be accumulated at the hanging mercury drop electrode (HMDE) surface and afterwards stripped off by applying a potential scan [18]. The introduc- 


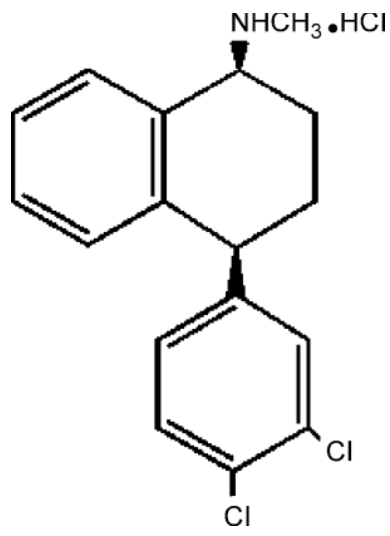

Fig. 1. Chemical structure of sertraline.

tion of high scan rate voltammetric techniques like square wave voltammetry allows increasing the sensitivity of AdSV even further [19,20].

In the present paper, the possibility of combining a flow injection system with a voltammetric detector, applying adsorptive stripping square wave voltammetry, for the determination of STR in a pharmaceutical preparation was explored. Using the HMDE flow cell developed by Rodrigues et al. [21], it was possible to determine STR in flow at a high sample rate and reduced costs, opening the possibility to compete with the chromatographic methods generally used for this analysis.

\section{Experimental}

\subsection{Apparatus}

Voltammetric measurements were performed using an $\mathrm{Au}-$ tolab PGSTAT12 (Metrohm-Eco Chemie), controlled by a PC with the GPES 4.9 software (Metrohm-Eco Chemie), linked to a Metrohm 663VA stand containing a three-electrode cell (all Metrohm). This cell consisted of a multimode mercury working electrode, used in the static mercury drop position, an $\mathrm{Ag} / \mathrm{AgCl} / \mathrm{KCl} 3 \mathrm{~mol} \mathrm{~L}^{-1}$ reference electrode, and a glassy carbon auxiliary electrode.

The above described equipment was used as a detector in a single manifold flow injection analysis (FIA) system. Samples and standards were introduced $\left(V_{\text {inj }}=500 \boldsymbol{\mu L}\right)$ into the carrier stream through a six-port Rheodyne 5041 injection valve. The solutions were transported through the FIA system by a Gilson Minipuls 3 peristaltic pump, using PTFEcarrier tubing (i.d. $0.8 \mathrm{~mm}$ ), to the voltammetric HMDE cell.

In the voltammetric flow cell used in this work [21], the glass capillary of the mercury electrode is inserted into a PTFE adapter head and the flow is directed towards the mercury drop. This adapter head allows a reproducible repositioning of the glass capillary to be made whenever the flow cell is dismounted and then remounted. The adapter head together with the reference electrode and the counter electrode are contained in a glass cell, which is filled with the solution used as the carrier stream. A drain ensures a constant level of solution to be maintained during the work.

\subsection{Reagents}

A sertraline hydrochloride standard was kindly offered by Pfizer Inc. All other chemicals were of analytical grade quality. Deionised water (conductivity $<0.1 \mathbf{\mu S ~ c m}^{-1}$ ) was used throughout. The supporting electrolyte was a borate buffer (pH 8.2; $0.1 \mathrm{M})$.

Sertraline stock solutions $\left(10^{-4} \mathrm{~mol} \mathrm{~L}^{-1}\right)$ were prepared in methanol and stored at $4{ }^{\circ} \mathrm{C}$. These stock solutions were further diluted with electrolyte solution or with an electrolyte-methanol mixture.

\subsection{Pharmaceutical preparation}

Five tablets of Zolof (50 mg STR/tablet) were weighed and finely powdered. About $20 \mathrm{mg}$ of this powder was dissolved in $25 \mathrm{~mL}$ of methanol with the help of an ultrasonic bath. The resulting solution was then filtered to eliminate non-dissolved excipient and diluted about 200 times, with an electrolyte-methanol mixture, to obtain the required STR concentration. Quantification of STR was carried out by the standard addition method.

\section{Results and discussion}

In the development of the flow injection voltammetric system for the determination of STR, it was important to consider the results previously reported by Vela et al. [17]: STR adsorbs at the HMDE and presents a reduction peak at about $-1.8 \mathrm{~V}$ versus $\mathrm{AgCl} / \mathrm{Ag}$ in an electrolyte of $\mathrm{pH}$ 8.2.

Then, the influence of deoxygenation on the determination of STR was studied and it was observed that the dissolved oxygen did not interfere when a preconcentration of $30 \mathrm{~s}$ ( $E_{\text {acc: }}:-1.2 \mathrm{~V}$ versus $\mathrm{AgCl} / \mathrm{Ag}$ ) was applied, even when low scan rates and concentrations $\left(10^{-7} \mathrm{~mol} \mathrm{~L}^{-1}\right)$ were used (Fig. 2). The possibility of performing the measurements without the previous deoxygenation step allows a significant reduction of the analysis' time and the possibility of including the HMDE in a flow injection system, without prior or on-line oxygen removal, which leads to an increase in sample rate.

Based on the previous results, a flow injection system was developed, in which the adsorption step at the HMDE was performed, while the sample solution slug was flowing through the adapter head containing the mercury electrode. The square wave voltammetric scan was also performed under flow conditions and no perturbation of the voltammetric signal due to the flow of the electrolyte was observed. The length of the tube between the injection valve and the HMDE was kept as short as possible in order to minimise dispersion of the injected sample, and to keep the injected slug as intact as possible. In such conditions, it was possible to obtain a 

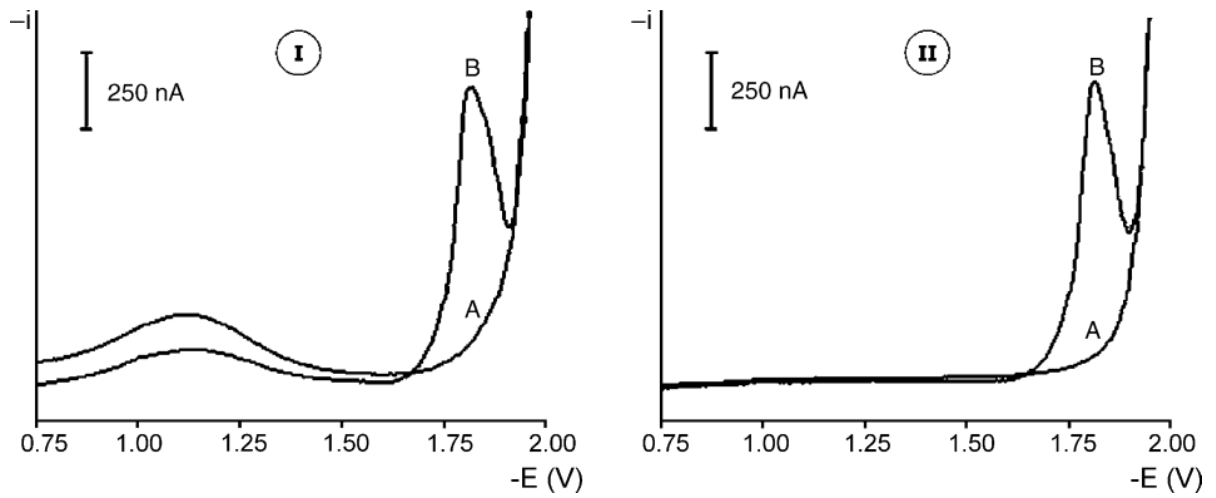

Fig. 2. Influence of dissolved oxygen on the voltammetric determination of STR. STR concentration: (A) 0 mol L ${ }^{-1}$ and (B) $5 \times 10^{-7} \mathrm{~mol} \mathrm{~L}^{-1}$. Deoxygenation time: (I) $0 \mathrm{~s}$ and (II) $600 \mathrm{~s}$. Eacc: $-1.2 \mathrm{~V}$, tacc: $30 \mathrm{~s}, f: 50 \mathrm{~Hz}, E_{\text {step }}: 5 \mathrm{mV}$ and $E_{\text {ampl: }} 25 \mathrm{mV}$.

constant concentration sample zone passing at the electrode with a thickness depending on the injection volume (fixed at $500 \mathrm{\mu L}$ ) and on the flow rate. At higher flow rates, the period of constant concentration throughput becomes smaller, reducing the time available for the adsorption and voltammetric scan steps to be performed.

Addition of methanol to the sample solution led to a significant increase in peak current intensity $\left(i_{\mathrm{p}}\right)($ Fig. 3) and precision. However, for methanol contents higher than $10 \%$ (v/v) no further increase in $i_{\mathrm{p}}$ and poor peak definitions were obtained, reason why sample solutions were prepared in electrolyte solution containing $10 \%$ (v/v) methanol.

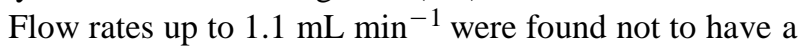
great effect on the adsorption process. For higher flow rates, $i_{\mathrm{p}}$ decreased significantly, probably due to the removal of adsorbed species at the electrode by the flow of electrolyte. The adopted flow rate was $1.1 \mathrm{~mL} \mathrm{~min}^{-1}$, a good compromise between high sample rate and sensitivity. Using this flow rate,

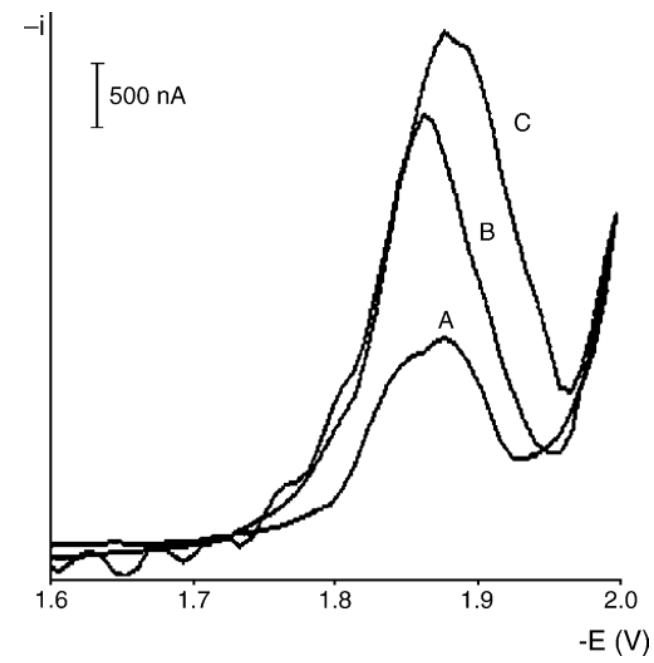

Fig. 3. Flow injection voltammograms of a $5 \times 10^{-6} \mathrm{~mol} \mathrm{~L}^{-1} \mathrm{STR}$ solution in pH 8.2 buffer, containing: (A) $0 \%$, (B) $5 \%$ and (C) $10 \%$ (v/v) methanol. $E_{\text {acc: }}-1.2 \mathrm{~V}, t_{\mathrm{acc}}: 5 \mathrm{~s}, f: 10 \mathrm{~Hz}, E_{\text {step }}: 8 \mathrm{mV}, E_{\text {ampl: }}: 45 \mathrm{mV}$ and flow rate: $1.1 \mathrm{mLmin}^{-1}$. an injection volume of $500 \boldsymbol{\mu L}$ and a tube length of $50 \mathrm{~cm}$ between the injector and the detector, a constant concentration profile between 15 and $40 \mathrm{~s}$ after injection was obtained. Both the adsorption step and the voltammetric scan must be performed within this time interval.

The applied accumulation potential was the same used in batch conditions $(-1.2 \mathrm{~V}$ versus $\mathrm{AgCl} / \mathrm{Ag})$. The effect of the accumulation time is depicted in Fig. 4, with a marked increase in $i_{\mathrm{p}}$ when the accumulation time is increased from 0 to $6 \mathrm{~s}$. For longer accumulation times (up to $10 \mathrm{~s}$ ) $i_{\mathrm{p}}$ levels off, possibly due to saturation of the drop coverage, to competitive adsorption or to removal of species by the flow.

In order to obtain maximum sensitivity and reproducibility/repeatability, several other SWAdSV parameters, the frequency, the pulse step and the pulse amplitude, were studied and optimised. These parameters are interrelated and have a combined impact on the signal. In order to find the optimum conditions, experiments were carried out using different frequencies $(10-100 \mathrm{~Hz})$, pulse steps $(1-10 \mathrm{mV})$ and pulse amplitudes $(5-40 \mathrm{mV})$. From these studies, the optimum conditions for the determination of STR were established as $f=10 \mathrm{~Hz}, E_{\text {step }}=5 \mathrm{mV}$ and $E_{\mathrm{ampl}}=25 \mathrm{mV}$. Using these optimised conditions, a good linear correlation between $i_{\mathrm{p}}$ and STR concentration was obtained for the concentration interval between $0.20 \times 10^{-6}$ and $1.20 \times 10^{-6} \mathrm{~mol} \mathrm{~L}^{-1}$

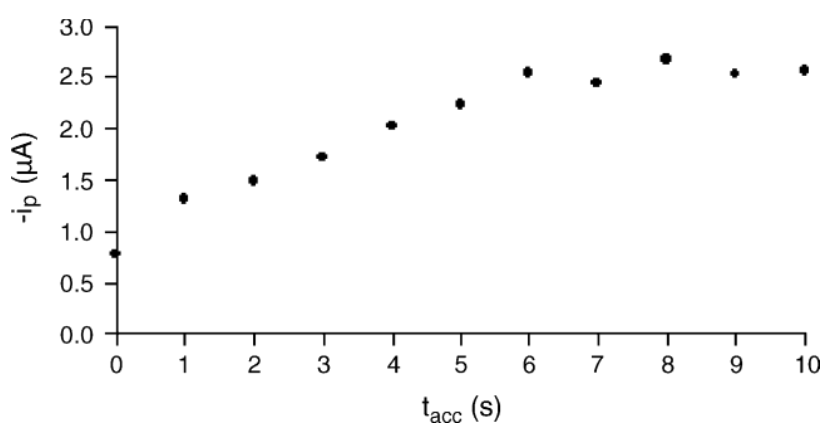

Fig. 4. Variation of $i_{\mathrm{p}}$ with accumulation time for a $2 \times 10^{-6} \mathrm{~mol} \mathrm{~L}^{-1}$ STR solution. 


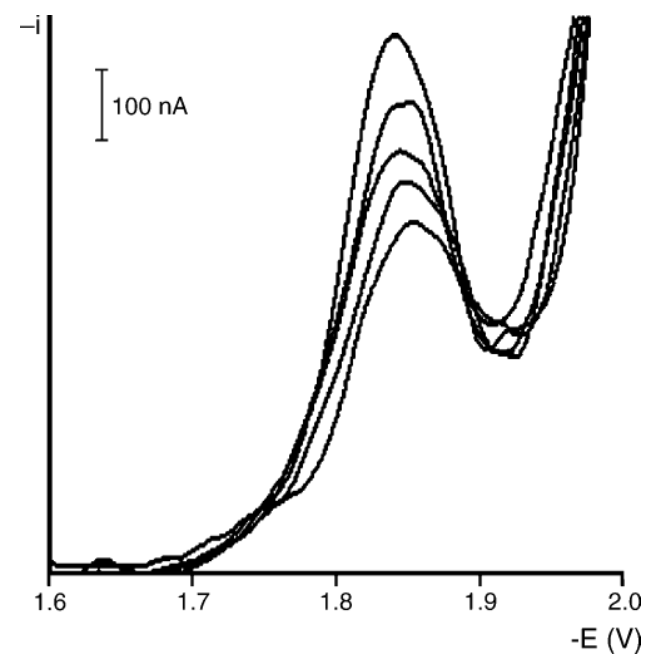

Fig. 5. Typical flow injection voltammograms obtained in the quantification of STR in a pharmaceutical preparation (Zoloft) using the standard addition method. Standard additions of STR $\left(10^{-7} \mathrm{~mol} \mathrm{~L}^{-1}\right): 0,3.00,4.00,5.00$ and 6.00. $E_{\text {acc: }}-1.2 \mathrm{~V}, t_{\text {acc: }}: 15 \mathrm{~s}, f: 10 \mathrm{~Hz}, E_{\text {step }}: 5 \mathrm{mV}, E_{\text {ampl: }}: 25 \mathrm{mV}$ and flow rate: $1.1 \mathrm{mLmin}^{-1}$.

$\left(i_{\mathrm{p}}=0.939 c_{\mathrm{STR}}+2.05 \times 10^{-8} ; r=0.995, n=5\right)$. The limits of detection (LOD) and quantification (LOQ) were calculated using this calibration curve [22], and were found to be $1.5 \times 10^{-7}$ and $5.0 \times 10^{-7} \mathrm{~mol} \mathrm{~L}^{-1}$, respectively. Up to 70 samples could be analysed per hour with good precision (R.S.D. $=2.5 \%(n=10)$ at a $1.0 \times 10^{-6} \mathrm{~mol} \mathrm{~L}^{-1}$ concentration level).

At the moment, the only commercially available pharmaceutical on the Portuguese market that contains STR is Zoloft (Pfizer Inc.). The labelled value of STR per tablet is $50 \mathrm{mg}$. In order to evaluate the adequacy of the developed method, it was applied to the determination of STR in this pharmaceutical by the standard addition method. In Fig. 5, typical flow injection voltammograms corresponding to the quantification of STR in Zoloft are shown. A mean result of $48.3 \pm 2.4 \mathrm{mg} /$ tablet $(n=3)$ was obtained, which conforms to the labelled value $(50 \mathrm{mg} /$ tablet $)$.

\section{Conclusions}

In this work, a method for the determination of STR was developed, using adsorptive voltammetry and a HMDE flow cell. The method can be applied as a good alternative to chromatographic methods for the determination of STR in pharmaceutical preparations. It has the advantages of significantly reducing the analysis' time and costs and obtaining a high sample rate.
Further investigation is undertaken in order to determine STR in biological samples using on-line sample extraction and the HMDE flow cell as a HPLC detector.

\section{Acknowledgements}

The authors thank Pfizer Inc. for providing the Sertraline hydrochloride standard. One of the authors (Henri P.A. Nouws) thanks the PRODEP III program for his Ph.D. grant.

\section{References}

[1] C. Duverneuil, G.L. de la Grandmaison, P. de Mazancourt, J.C. Alvarez, Ther. Drug Monit. 25 (2003) 565-573.

[2] K. Titier, N. Castaing, E. Scotto-Gomez, F. Pehourcq, N. Moore, M. Molimard, Ther. Drug Monit. 25 (2003) 581-587.

[3] C. Frahnert, M.L. Rao, K. Grasmader, J. Chromatogr. B 794 (2003) 35-47.

[4] P. Dallet, L. Labat, M. Richard, M.H. Langlois, J.P. Dubost, J. Liq. Chromatogr. Related Technol. 25 (2002) 101-111.

[5] G. Tournel, N. Houdret, V.Hedouin, M. Deveaux, D. Gosset, M. Lhermitte, J. Chromatogr. B 761 (2001) 147-158.

[6] G. Casamenti, R. Mandrioli, C. Sabbioni, F. Bugamelli, V. Volterra, M.A. Raggi, J. Liq. Chromatogr. Related Technol. 23 (2000) 10391059 .

[7] E. Lacassie, J.M. Gaulier, P. Marquet, J.F. Rabatel, G. Lachatre, J. Chromatogr. B 742 (2000) 229-238.

[8] A. Lucca, G. Gentilini, S. Lopez-Silva, A. Soldarini, Ther. Drug Monit. 22 (2000) 271-276.

[9] L. Labat, M. Deveaux, P. Dallet, J.P. Dubost, J. Chromatogr. B 773 (2002) 17-23.

[10] T. Buzinkaiova, J. Polonsky, Electrophoresis 21 (2000) 2839-2841.

[11] D. Rogowsky, M. Marr, G. Long, C. Moore, J. Chromatogr. B 655 (1994) 138-141.

[12] M.A. Martinez, C.S. de la Torre, E. Almarza, J. Anal. Toxicol. 26 (2002) 296-302.

[13] M.A. Martinez, C.S. de la Torre, E. Almarza, J. Anal. Toxicol. 27 (2003) 8A.

[14] K.M. Kim, B.H. Jung, M.H. Choi, J.S. Woo, K.J. Paeng, B.C. Chung, J. Chromatogr. B 769 (2002) 333-339.

[15] C.B. Eap, G. Bouchoux, M. Amey, N. Cochard, L. Savary, P. Baumann, J. Chromatogr. Sci. 36 (1998) 365-371.

[16] L.I. Bebawy, N. El-Kousy, J.K. Suddik, M. Shokry, J. Pharm. Biomed. Anal. 21 (1999) 133-142.

[17] M.H. Vela, M.B. Quinaz Garcia, M.C.B.S.M. Montenegro, Fresenius J. Anal. Chem. 369 (2001) 563-566.

[18] J. Wang, Stripping Analysis: Principles, Instrumentation and Applications, VCH Publishers, Deerfield Beach, USA, 1985, pp. 61-63.

[19] L. Ramaley, J.A. Dalziel, W.T. Tan, Can. J. Chem. 59 (1981) 3334 3340 .

[20] A.A. Barros, J.A. Rodrigues, P.J. Almeida, P.G. Rodrigues, A.G. Fogg, Anal. Chim. Acta 385 (1999) 315-323.

[21] J.A. Rodrigues, A.A. Barros, P.J. Almeida, P.G. Rodrigues, A.G. Fogg, Anal. Chim. Acta 449 (2001) 119-127.

[22] J.N. Miller, J.C. Miller, Statistics and Chemometrics for Analytical Chemistry, fourth ed., Prentice Hall, Harlow, 2000, pp. 120-123. 